\title{
EFFECT OF MULBERRY LEES ADDITION ON DOUGH MIXING CHARACTERISTICS AND THE QUALITY OF MULBERRY TOAST
}

\author{
Jean-Yu Hwang \\ Department of Food Science and Technology, Chung-Hwa University of Medical Technology, Tainan, Taiwan,R.O.C. \\ Wen-Chieh Sung \\ Department of Hotel \& Restaurant Management, Chia Nan University of Pharmacy and Science, Tainan, Taiwan, R.O.C. \\ Yung-Shin Shyu \\ Department of Baking Technology and Management, Kaoushang Hospitality College, Taiwan, R.O.C., \\ tristar@mail.nkhc.edu.tw
}

Follow this and additional works at: https://jmstt.ntou.edu.tw/journal

Part of the Life Sciences Commons

\author{
Recommended Citation \\ Hwang, Jean-Yu; Sung, Wen-Chieh; and Shyu, Yung-Shin (2008) "EFFECT OF MULBERRY LEES ADDITION ON DOUGH \\ MIXING CHARACTERISTICS AND THE QUALITY OF MULBERRY TOAST," Journal of Marine Science and Technology. \\ Vol. 16: Iss. 2, Article 3. \\ DOI: $10.51400 / 2709-6998.2021$ \\ Available at: https://jmstt.ntou.edu.tw/journal/vol16/iss2/3 \\ This Research Article is brought to you for free and open access by Journal of Marine Science and Technology. It has been \\ accepted for inclusion in Journal of Marine Science and Technology by an authorized editor of Journal of Marine Science and \\ Technology.
}




\title{
EFFECT OF MULBERRY LEES ADDITION ON DOUGH MIXING CHARACTERISTICS AND THE QUALITY OF MULBERRY TOAST
}

\author{
Jean-Yu Hwang*, Wen-Chieh Sung**, and Yung-Shin Shyu***
}

\begin{abstract}
Key words: mulberry lees, Scanning Electron Microscopy (SEM), Differential Scanning Calorimetry (DSC), fermentation, dough.
\end{abstract}

\begin{abstract}
This study evaluated the effects of $10 \%-30 \%$ mulberry lees addition on the dough mixing characteristics and the quality of mulberry toast. Farinogram shows the addition of $30 \%$ mulberry lees had the shortest departure time and weakest gluten strength. Peak viscosity, pasting temperature and final viscosity of Rapid Visco Analyser pasting profiles decreased as the addition of mulberry lees increase. Dough with $10 \%$ and $20 \%$ lees addition had a similar dough expansion rate to that of the control. However, the fermentation rate of the dough with $30 \%$ mulberry lees was obviously much slower. The ice-melting onset temperature, peak temperature and enthalpy of Differential Scanning Calorimetry (DSC) decrease with the addition of mulberry lees. A scanning electron microscopy was used to study changes in structure of bread dough that had added mulberry lees and fermented for 240 minutes. Starch granules of the control dough appeared to be coated within a compact gluten matrix that retained its integrity after final proof. When the dough was added with $30 \%$ mulberry lees, the appearance of the dough altered from a loose and discontinuous protein matrix to an un-solidified and open structure after final proof. Mulberry lees could be added to bread dough at a level of less than $20 \%$ without significant reducing its bread volume and quality.
\end{abstract}

\section{INTRODUCTION}

High level of serum cholesterol, triglycerides, and plasma low-density lipoprotein cholesterol have been clearly identified as important risk factors to atherosclerosis and coronary heart disease. Mulberry (Morus alba L.) has gained much attention due to their antioxidant activity in retardation of atheroscleros-

Paper submitted 11/22/06; accepted 03/27/07. Author for correspondence: Yung-Shin Shyu (e-mail: tristar@mail.nkhc.edu.tw).

*Department of Food Science and Technology, Chung-Hwa University of Medical Technology, Tainan, Taiwan, R.O.C.

**Department of Hotel \& Restaurant Management, Chia Nan University of Pharmacy and Science, Tainan, Taiwan, R.O.C.

***Department of Baking Technology and Management, Kaoushang Hospitality College, Taiwan, R.O.C. tic lesion development [5-7, 10, 12, 13, 17, 19]. Anthocyanins [9], vitamin $\mathrm{C}$, flavonoids [7] of mulberry could reduce the incidence of heart diseases, such as atherosclerosis, through their antioxidant activity. Mulberry fruit, leaves, bark and branches have used in Chinese medicine to treat fever, facilitate discharge of liver, protect the liver damage, strengthen joint, and lower blood pressure [26]. Mulberry fruit also could be processed into juice, soft drink, jam, and wine [16] in food industry. Mulberry lees are the straining leftover of mulberry wine. They are also rich in dietary fiber and supply valuable vitamins and minerals. Plenty of beneficial effects of dietary fiber have been extensively studied [4,23]. The deterioration in bread quality (loaf volume and absorption) is prior due to inferior mixing characteristics in now before new methods and ingredients were developed. Mixing tolerance is an important quality parameter for the baker. Overall, it may be possible to make acceptable bread from any flour by using rigidly controlled conditions. However, if bread quality deteriorates markedly with only small changes in mixing or fermentation, the baker could have major problem in maintaining a consistent product [3]. In this study, mulberry lees were added to bread dough. Effects of adding mulberry lees on dough mixing characteristics, dough expansion, microstructures, and baking test were investigated. The results of the present study could be used for bakery industry and help the mulberry wine maker for easy the problem of leftover.

\section{MATERIALS AND METHODS}

\section{Materials}

Mulberry lees, used in this study were a leftover of mulberry wine, were obtained from National Pingtung University of Science and Technology (Pingtung, Taiwan). Bread flours were purchased from Uni-President Enterprises Corporation (Tainan, Taiwan). Shortening used in this study were purchased from Namchow group (Taoyuan, Taiwan). Instant dry yeast was purchased from Yung Cheng Industries Ltd. (Taipei, Taiwan).

\section{Methods}

\section{1) Physicochemical Properties of Flour and Dough Mixing}

Farinographs (C.W. Brabender Instruments, Inc., South Hackensack, NJ) were run at $30^{\circ} \mathrm{C}$ and the bread dough was made from $300 \mathrm{~g}$ bread flour and added water to center at 500 
Brabender Units by adjusting dough water content. It is used to access flour water absorption, arriving time, departure time, stability, peak time according to AACC Method 54-21 [1]. Therefore, the amounts of water added to $0 \%, 10 \%, 20 \%$, and $30 \%$ mulberry dough were $64 \%, 59 \%, 52 \%$, and $46 \%$, respectively.

The baking formula, based on baker's percentage, was as follows, $100 \%$ flour, $10 \%$ sugar, $1.5 \%$ salt, $1 \%$ salt, $1 \%$ dry yeast, $4 \%$ shortening, 0\%-30\% mulberry lees, and water as determined from a farinograph absorption test. All the ingredients except shortening are combined and mixed into a dough. The shortening was added after the dough was developed. Vertical mixer (Jen Dah Food machinery, Chiayi, Taiwan) attached with dough arm is used to mix experimental dough to optimum development. Dough is divided and rounded into a ball shape. The molded dough was fermented in final proof room for a further 50 minutes at a temperature of $38^{\circ} \mathrm{C}$ and relative humidity of $85^{\circ} \mathrm{C}$. The fermented dough was baked in a electric oven (Jen Dah Food machinery, Chiayi, Taiwan) at 150 ${ }^{\circ} \mathrm{C} / 180^{\circ} \mathrm{C}$ for 25 minutes. Loaf volume was measured immediately after baking by a rapeseed displacement method.

The viscoamylographs of the flours were determined using a Rapid Visco Analyzer model 3D (RVA) (Newprot Scientific Pty Ltd., Warriewood, Australia) following the method of Whalen et al. [25]. The RVA 3D were operated with $3.5 \mathrm{~g}$ bread flour, 25 $\mathrm{ml}$ water, and $0 \%, 10 \%, 20 \%$, and $30 \%$ mulberry lees addition, respectively. The temperature profile included a $1 \mathrm{~min}$ isothermal step at $50^{\circ} \mathrm{C}$, a linear temperature increase to $95^{\circ} \mathrm{C}$ in $3.75 \mathrm{~min}$, a holding step $\left(2.5 \mathrm{~min}\right.$ at $\left.95^{\circ} \mathrm{C}\right)$, a cooling step $(3.75$ min) with a linear temperature decrease to $50^{\circ} \mathrm{C}$. The RVA pasting parameters of peak viscosity, trough, breakdown, peak time, pasting temperature, final viscosity and setback were measured in triplicate.

\section{2) $\mathrm{pH}$ Measurement}

Five grams dough was added 45 grams of distilled water. It was blended and filtered with Whatman No. 1 filter paper before $\mathrm{pH}$ analysis (Corning Scientific Co., NY). A combination electrode, standardized between $\mathrm{pH} 4.0$ and 7.0 and attached to a pH/ion analyzer (Corning Scientific Co., NY), was inserted into the strained liquid every 20 minutes.

\section{3) Dough Expansion}

Dough expansion test followed the method of Sangnark and Noomhorm [21] with slight modification. Dough after mixing was divided into 50 gram pieces and rounded. Dough samples were inserted into a $250 \mathrm{ml}$ graduated cylinder. Cylinders were placed in a cabinet at a temperature of $28^{\circ} \mathrm{C}$ and relative humidity of 75 for 240 minutes. Dough volume was recorded every 20 minutes.

\section{4) Thermal Characteristics}

Ice melting temperature was determined using a modulated DSC 2010 differential scanning colorimeter (TA instrument, New Castle, USA) following the methods of Biliaderis et al. [2]. Dough samples (10 mg) were placed in aluminum crucibles and the crucible was hermetically sealed. An empty crucible was
Table 1. Effect of mulberry lees addition on dough Farinography parameters ${ }^{1}$

\begin{tabular}{lcccc}
\multicolumn{1}{c}{ rameters } & Control & $10 \%$ & $20 \%$ & $30 \%$ \\
\hline Water absorption (\%) & 64 & 59 & 52 & 46 \\
Arrival time (min) & 0.5 & 1.0 & 1.5 & 1.5 \\
Peak time (min) & 2.0 & 2.5 & 4.0 & 6.0 \\
Departure time (min) & 30.0 & 19.0 & 17.5 & 14.5 \\
Mixing stability (min ) & 29.5 & 18.0 & 16.0 & 13.0 \\
\hline
\end{tabular}

${ }^{1} \overline{\text { All values were a mean of } 2 \text { replications with } 2 \text { sub-samples per replication }}$.

used as reference. The ice melting temperature parameters of To (onset), Tp (peak), Tc (conclusion), and enthalpy ( $\triangle \mathrm{H}, \mathrm{J} / \mathrm{g})$ were determined. The dough was heated from $-50^{\circ} \mathrm{C}$ to $20^{\circ} \mathrm{C}$ at $5^{\circ} \mathrm{C} / \mathrm{min}$ and carried out all experiments in triplicate.

\section{5) Texture Properties of Bread}

Breads were sealed in $1 \mathrm{Kg}$ polyethylene (PE) bags after baking for 1 hour and held at room temperature $\left(25^{\circ} \mathrm{C}\right)$ for further testing. Breads were sliced into $3 \times 3 \times 3 \mathrm{~cm}^{3}$ crumbs using a standard bread slice. Hardness of breads were tested with the TA.XT2 Texture Anaylyzer (Haslemere, England) and a $75 \mathrm{~mm}$ diameter cylinder probe according to the methods of Fiszman et al. [8]. Texture profile analysis (TPA) was conducted with a test speed of $5.0 \mathrm{~mm} / \mathrm{s}$. Calibration distance for the probe was $35.0 \mathrm{~mm}$.

\section{6) Scanning Electron Microscopy}

Dough was examined during fermentation followed the methods of Kim et al. [15]. Dough samples were freeze vacuum dried at $-50^{\circ} \mathrm{C}$ for 24 hours. Freeze-dried dough was cut with a razor blade and mounting onto brass stubs using double-sided carbon conductive adhesive tape. A gold coat (15nm thick) was then applied using a sputter coater (Giko Engineering, IB model). Samples were examined at $25 \mathrm{keV}$ using a Hitachi S-2500 Scanning Electron Microscopy (Tokyo, Japan).

\section{7) Statistical Analysis}

The data were analyzed statically with the Statistic Analysis System [22]. ANOVA and Duncan's test used to test the significance difference of each group. A value of $p<0.05$ was considered significant.

\section{RESULTS AND DISCUSSION}

\section{Dough Properties}

Table 1 shows water absorption of farinograms decreases with the addition of mulberry lees. The moisture content of mulberry lees is $91.2 \%$, which would be partial absorbed by bread flour before water addition and dough mixing process. The farinograms also show that the arriving time and peak time parameters increased with the addition of mulberry lees. The addition of $30 \%$ mulberry lees dough is too weak, as reflected 
Table 2. Effect of mulberry lees addition on dough RVA pasting properties ${ }^{1}$.

\begin{tabular}{lcccc}
\hline & Control & $10 \%$ & $20 \%$ & $30 \%$ \\
\hline Peak viscosity (RVU) & $241.1^{\mathrm{c}}$ & $246.4^{\mathrm{bc}}$ & $249.2^{\mathrm{ab}}$ & $254.5^{\mathrm{a}}$ \\
Trough (RVU) & $164.3^{\mathrm{a}}$ & $157.0^{\mathrm{b}}$ & $151.9^{\mathrm{bc}}$ & $150.9^{\mathrm{c}}$ \\
Breakdown (RVU) & $76.7^{\mathrm{d}}$ & $89.4^{\mathrm{c}}$ & $97.2^{\mathrm{b}}$ & $103.6^{\mathrm{a}}$ \\
Peak time (min) & $6.3^{\mathrm{a}}$ & $6.3^{\mathrm{a}}$ & $6.3^{\mathrm{a}}$ & $6.2^{\mathrm{a}}$ \\
Pasting temp ( $\left.{ }^{\circ} \mathrm{C}\right)$ & $71.7^{\mathrm{a}}$ & $68.3^{\mathrm{b}}$ & $67.3^{\mathrm{b}}$ & $67.3^{\mathrm{b}}$ \\
Final viscosity (RVU) & $273.8^{\mathrm{a}}$ & $267.1^{\mathrm{b}}$ & $266.0^{\mathrm{b}}$ & $265.6^{\mathrm{b}}$ \\
Setback (RVU) & $109.5^{\mathrm{a}}$ & $110.1^{\mathrm{a}}$ & $114.1^{\mathrm{a}}$ & $114.7^{\mathrm{a}}$
\end{tabular}

${ }^{1}$ All values were a mean of 3 replications with 3 sub-samples per replication. Mean values with the different letter in the same column were significantly different $(\mathrm{p}<0.05)$

by short mixing stability and rapid departure time. Mulberry lees lack the gluten functionality will retard the development of bread dough. It is suggested the addition of water amount should decrease for making food products such as bread, cake and floured based noodles. Hamed et al. [11] reported sweet potato flour was added and the bread dough exhibited degrading the gluten network of bread dough. They proposed adding sweet potato flour to wheat flour will increase water absorption and decrease the peak time of farinogram. The increase water absorption as observed for sweet potato flour in their study might be due to the moisture content of sweet potato flour is less than $12 \%$, which would competes water with bread flour.

\section{Pasting Characteristics}

It was found addition of mulberry lees did not significantly affect peak time of RVA, except for trough, final viscosity and peak viscosity (Table 2). Trough and final viscosity of the RVA parameters decreased with addition of mulberry lees. When $30 \%$ of mulberry lees were added into flour, the peak viscosity of flour paste would increase. This was not surprising, since the mulberry lees contain $92 \%$ of moisture. As a consequence, more water go into the interior of starch granules even when the adding amounts was $10 \%$, induces the effect obviously. Apparently, starches of test groups swell more during gelatinization and pasting. The higher peak viscosity of test groups can be explained by higher water penetration into the starches and/or the dietary fiber of mulberry lees interacts with amylose and amylopectin to form hydrogen bonds. The addition of mulberry lees decreased the final viscosity of pasting curves by forming the hydrogen bonds between dietary fiber and starch polymers.

\section{Effect of Mulberry Lees on pH Change of Fermented Dough}

The $\mathrm{pH}$ of control group is highest (5.85). The decrease $\mathrm{pH}$ of control dough after fermentation was marked in comparison with that of test groups, whilst the $\mathrm{pH}$ of mulberry lees added dough was slightly decreased. Beyond 240 minutes of fermentation, $\mathrm{pH}$ of control dough decreased to 5.25 and led to a

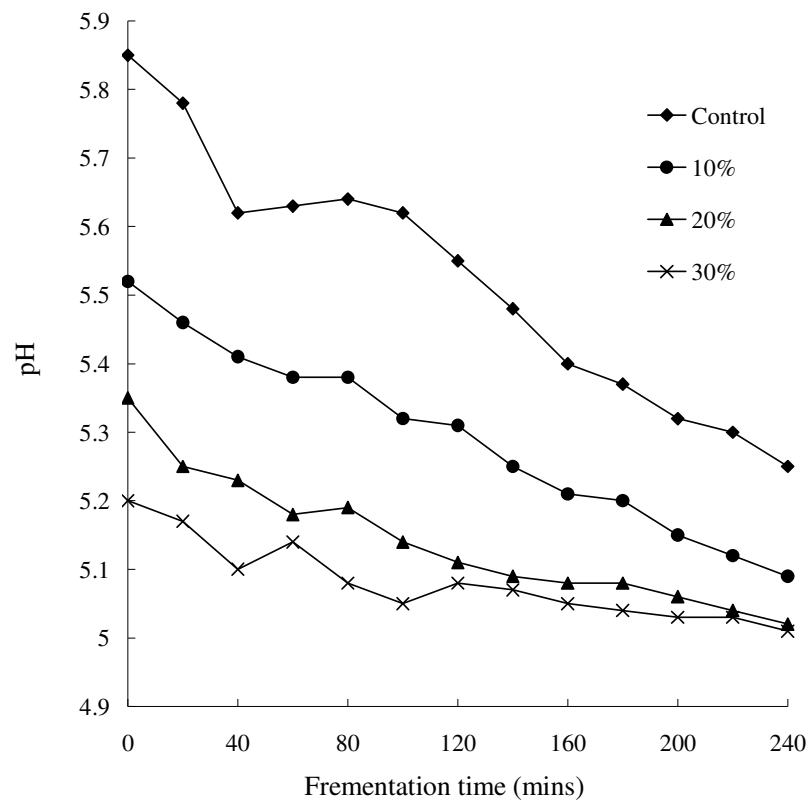

Fig. 1. Effect of mulberry lees addition on dough $\mathrm{pH}$ value during fermentation.

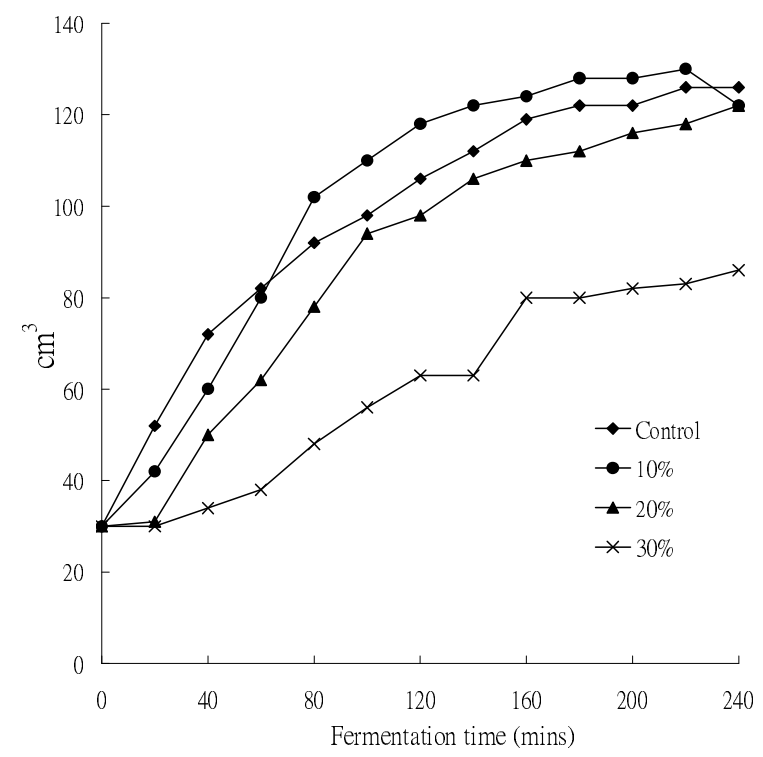

Fig. 2. Effect of mulberry lees addition on dough volume during fermentation.

reduction by $10.3 \%$. The $\mathrm{pH}$ of $30 \%$ mulberry lees addition decreased from 5.20 to 5.01 with a reduction of $3.7 \%$ at the end of fermentation. The $\mathrm{pH}$ of mulberry lees was 3.58. Thus, they also reduced the $\mathrm{pH}$ of $30 \%$ mulberry lees dough to 5.20 after mixing (Fig. 1). High level of mulberry lees addition will generate a lower $\mathrm{pH}$ dough and lower $\mathrm{pH}$ reduction after fermentation.

\section{Effect on Dough Expansion Test of Mulberry Lees Ad- dition}

As illustrated in Fig. 2, the dough volume increase of $10 \%$ 
Table 3. Effect of mulberry lees addition on dough differential scanning calorimetry (DSC) ice-melting onset temperature, peak temperature, enthalphy ${ }^{1}$ and loaf volume ${ }^{2}$.

\begin{tabular}{ccccc}
\hline $\begin{array}{c}\text { Mulberry } \\
\text { lees addi- } \\
\text { tion }\end{array}$ & $\begin{array}{c}\text { Onset T } \\
\left({ }^{\circ} \mathrm{C}\right)\end{array}$ & $\begin{array}{c}\text { Peak T } \\
\left({ }^{\circ} \mathrm{C}\right)\end{array}$ & $\begin{array}{c}\text { Enthalphy } \\
(\mathrm{J} / \mathrm{g})\end{array}$ & $\begin{array}{c}\text { Loaf vol- } \\
\text { ume }\left(\mathrm{cm}^{3}\right)\end{array}$ \\
\hline Control & -8.86 & -3.76 & 76.86 & $1996^{\mathrm{a}}$ \\
$10 \%$ & -8.90 & -4.30 & 73.89 & $2037^{\mathrm{a}}$ \\
$20 \%$ & -9.45 & -4.78 & 70.45 & $1869^{\mathrm{b}}$ \\
$30 \%$ & -11.04 & -5.25 & 65.25 & $1799^{\mathrm{b}}$ \\
\hline
\end{tabular}

${ }^{1}$ All values were a mean of 2 replications with 2 sub-samples per replication ${ }^{2}$ All values were a mean of 3 replications with 3 sub-samples per replication. Mean values with the different letter in the same column were significantly different $(\mathrm{p}<0.05)$

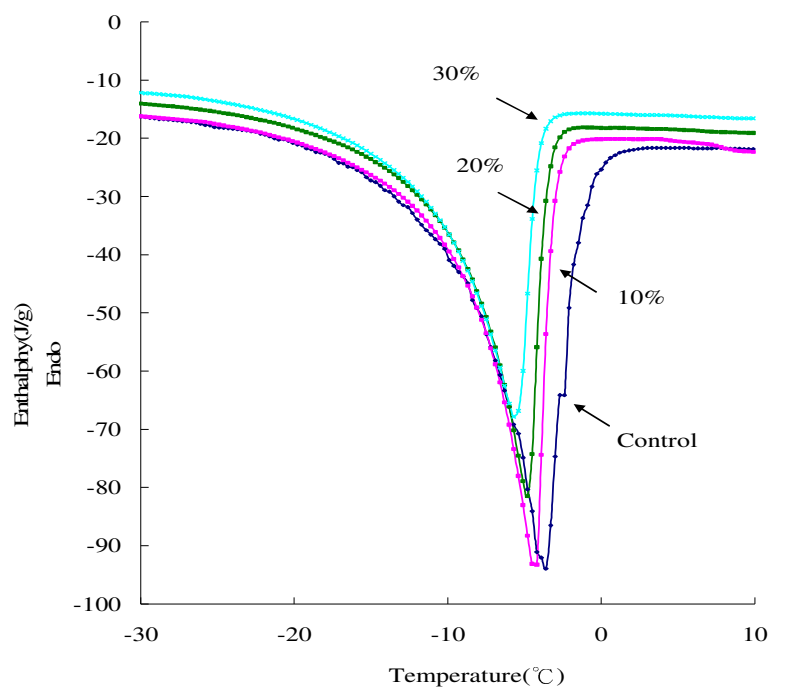

Fig. 3. Effect of mulberry lees addition on the dough ice melting temperature of Differential Scanning Calorimetry (DSC).

and $20 \%$ mulberry lees was similar to that of control group. However, the $30 \%$ addition of mulberry lees was obviously unable to retain the $\mathrm{CO}_{2}$ which generated by the yeast. This is possibly due to it contains less gluten and high amounts of mulberry lees will retard yeast growth. Dough made from the control, $10 \%$ and $20 \%$ mulberry lees addition had a faster volume increase within the first 150 minutes than $30 \%$ mulberry lees addition. Then, all those three groups induced a slow volume increase after 150 minutes fermentation time. Optimum growth $\mathrm{pH}$ of yeast is $\mathrm{pH} 4.0$ to $\mathrm{pH} 6.0$. The $\mathrm{pH}$ of $30 \%$ mulberry lees dough is around $\mathrm{pH} 5.0$ to $\mathrm{pH} 6.0$, so the yeast still can grow well in this $\mathrm{pH}$ range. The results in Fig. 1 and Fig. 2 show the effect on lowing $\mathrm{pH}$ of mulberry lees addition is not major factor for retard dough fermentation. Dough volume of $30 \%$ mulberry lees addition $\left(86 \mathrm{~cm}^{3}\right)$ explaining the low fermented dough volume could be mainly attributed to the diluted gluten content of the dough, so its final volume is significantly lower than other group $\left(122-126 \mathrm{~cm}^{3}\right)$.

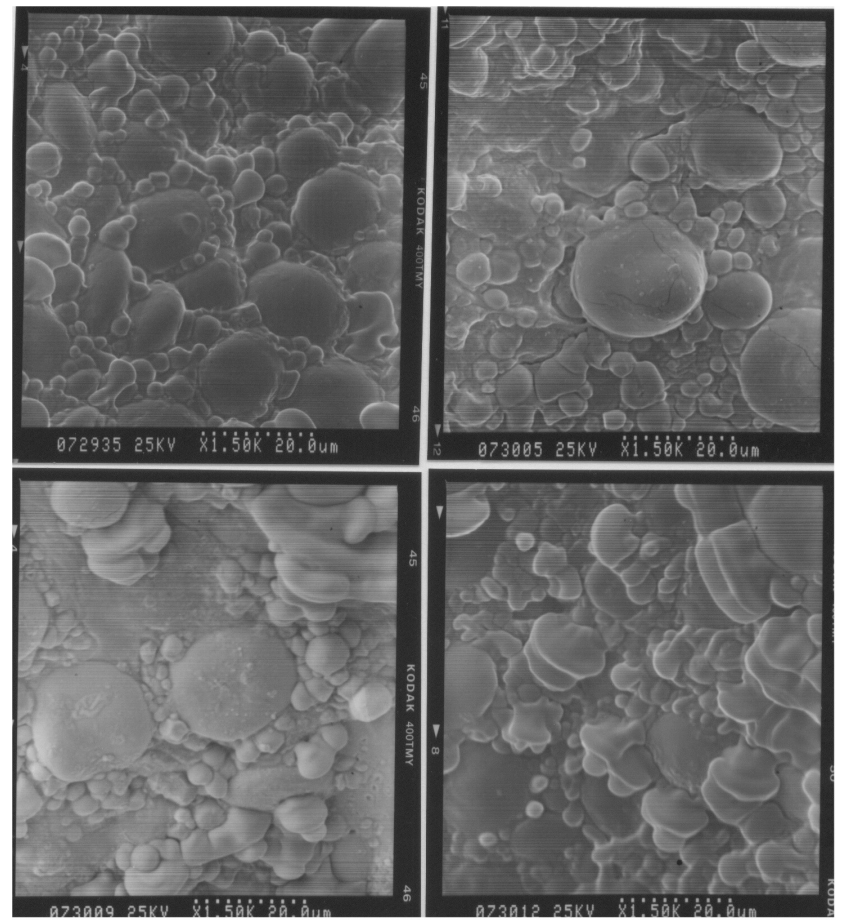

Fig. 4. Scanning electron micrographs of dough at completion mixing. (A) control dough (B) addition of $10 \%$ mulberry lees $(C)$ addition of $20 \%$ mulberry lees (D)addition of $30 \%$ mulberry lees. (x1500)

\section{Effect of Mulberry Lees Addition on Dough Thermal Characteristics}

Differential scanning calorimetry thermograms in all samples tested showed an endothermic peak around $0^{\circ} \mathrm{C}$ that was attributed to ice melting [24] and was therefore used to calculate the amount of freezable water. There was considerable variation in ice-melting temperatures and enthalpy among the control and test groups. The mean ice-melting onset temperature (To) was -8.86 for control. The ice-melting temperature of mulberry lees addition appeared lower than that of control after differential scanning calorimeter determination (Table 3). Ice-melting peak temperature (Tp) also decline by mulberry lees addition (Fig. 3). The $\triangle \mathrm{H}$ of ice-melting was $76.86 \mathrm{~J} / \mathrm{g}$ for the control group. The addition of $30 \%$ mulberry lees decreases the ice-melting enthalphy to $65.25 \mathrm{~J} / \mathrm{g}$ is postulated as a possible explanation for the amount of frozen dough of $30 \%$ mulberry lees addition has less free water than the other dough. It indicates the control dough had more freezable water associated with the melting curve than the dough containing mulberry lees $(30 \%)$.

\section{Effect of Mulberry Lees Addition on the Microstruc- ture of Dough}

Numerous starch granules of varying size were visible on the outer surface of gluten matrix. The control sample appears to be coated with a smooth protein film (arrow of Fig. 4A). As other researchers $[14,18,20]$ have reported previously, the dough of $10 \%$ mulberry lees also exhibited numerous starch granules were coated with an amorphous protein matrix, but some small 


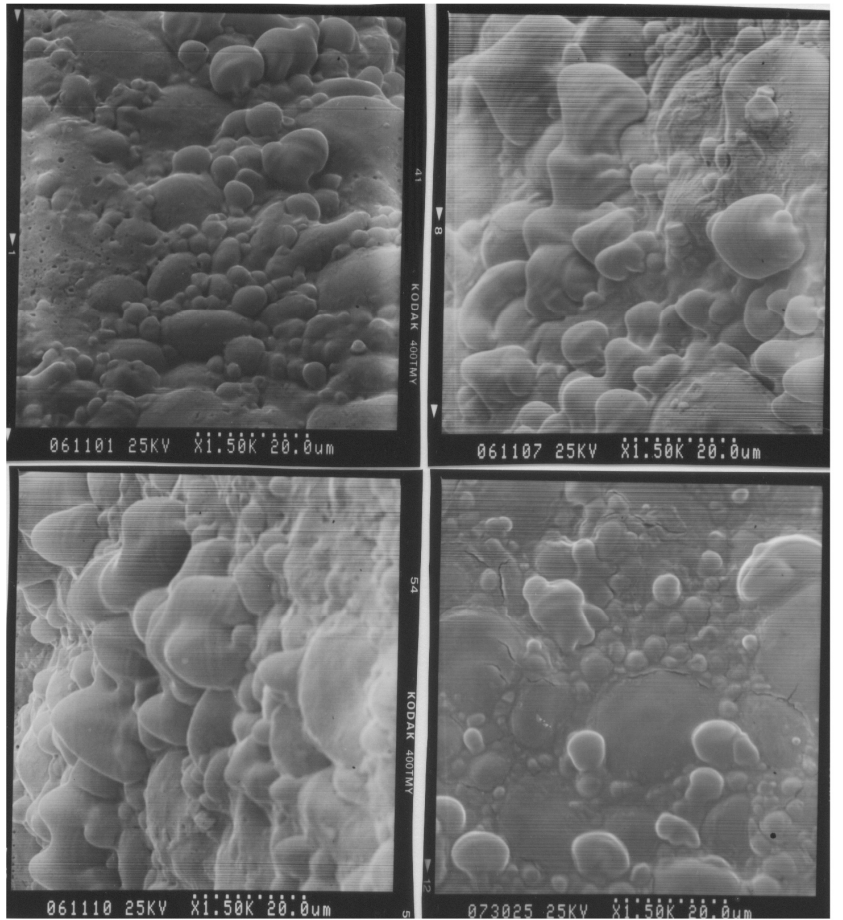

Fig. 5. Scanning electron micrographs of dough after first fermentation. (A) control dough (B) addition of $10 \%$ mulberry lees (C) addition of $20 \%$ mulberry lees (D) addition of $30 \%$ mulberry lees. $(\mathbf{x} 1500)$

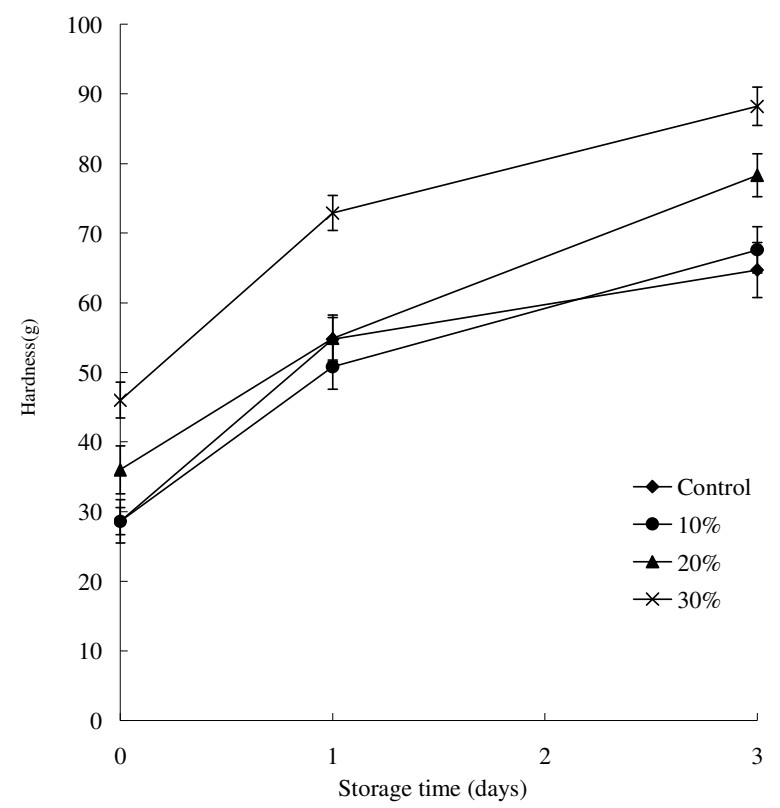

Fig. 6. Effect of mulberry lees addition on the hardness of toast.

holes and cracks were present on the surface (arrow of Fig. 4B). In contrast, Fig. 4D exhibited numerous small starch granules coated on an amorphous and less continuous protein matrix. The surface of the control dough (Fig. 5A) still appeared a smooth protein film, but the gluten matrix became less continuous and loose after fermentation. There were numerous small pores on it, which may be caused by the $\mathrm{CO}_{2}$ generated by the yeast (arrow of Fig. 5A). Fig. 5B shows the starch slightly

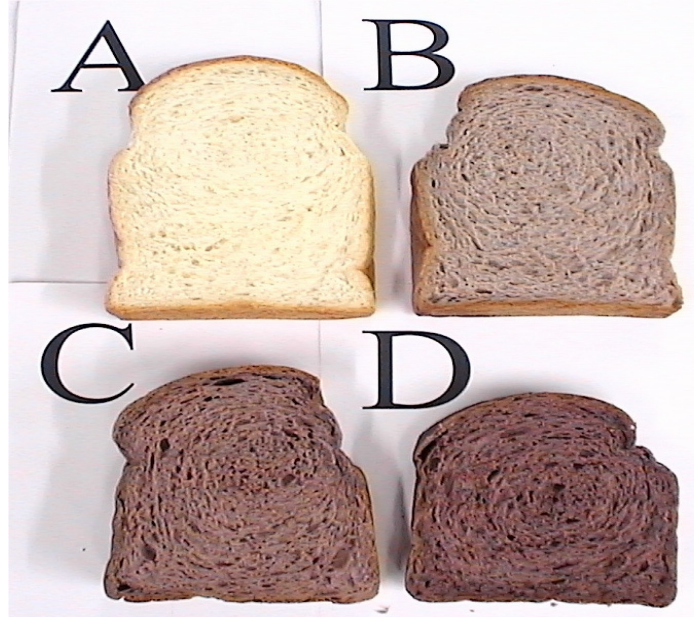

Fig. 7. Characteristics of bread made by various levels of mulberry lees addition (A) control bread (B) addition of $10 \%$ mulberry lees $(C)$ addition of $20 \%$ mulberry lees (D) addition of $30 \%$ mulberry lees.

attached to discontinuous protein film. After first fermentation, the dough surface of the $30 \%$ mulberry lees addition became smoother than the other groups.

The texture properties of bread crumb containing mulberry lees (up to $20 \%$ level) were not significantly ( $p>0.05$ ) different from the control bread on the day of baking. Addition of mulberry lees (less than $20 \%$ level) does not affect the hardness of breads containing mulberry lees (Fig. 6). Addition of $10 \%$ mulberry lees was effective in increasing loaf volume of bread. Loaf volume was significantly $(\mathrm{p}<0.05)$ decreased as the level of mulberry lees adding more than $20 \%$ (Table 3). Fig. 7 shows characteristics of bread made by various levels of mulberry lees addition. Crumb color of mulberry toast became darker and more purple with an increase in mulberry lees addition.

\section{CONCLUSION}

Mulberry lees addition ranging from 0 to $30 \%$ will increase the arrival time and peak time of dough Farinography parameters. It was shown the effects of mulberry lees addition reduced the mixing stability of Farinogram. The parameters, pasting temperature, peak viscosity and final viscosity of the RVA profile significantly decreased with mulberry lees addition. Addition of $30 \%$ mulberry lees has been proven to significantly retard the fermented rate of bread dough. The addition of $10-30 \%$ mulberry lees decreases dough $\mathrm{pH}$, but the decrease of $\mathrm{pH}$ is less than that of control sample after 240 minutes fermentation. It was shown the small spherical granules of wheat starches were slightly attached to discontinue protein matrix of mulberry lees dough. The structure of fermented dough appeared to be quite smooth and small starch granules was not readily apparent. Many small holes were apparent on the sample of fermented control dough that might be formed by penetration of $\mathrm{CO}_{2}$, which was generated by yeast fermentation. But those small holes were not apparent in the protein matrix of mulberry lees added dough after first fermentation. Our results suggest addition of $10 \%$ or $20 \%$ mulberry lees might be a good choice for the application of mulberry lees in bread making. 


\section{REFERENCES}

1. AACC: American Association of Cereal Chemists, Approved methods, AACC. St. paul, M.N., USA (1995).

2. Biliaderis, C. G., Maurice, T. J., and Vose, J. R., "Starch gelatinization phenomena studied by differential scanning calorimetry," Journal of Food Science, Vol. 45, pp. 1669-1674 (1980).

3. Canadian International Grains Institute (CIGI), "Bread making technology," Grains \& Oilseeds Handling, Marking, Processing, Vol. 2, Winnipeg, Manitoba, Canada, pp.655-696 (1993).

4. Champ, M., Guillon, F., "Sturctural and physical properties of dietary fibres, and consequences of processing on human physiology," Food Research International, Vol. 33, pp. 233-245 (2000).

5. Chen, C. C., Liu, L. K., Hsu, J. D., Huang, H. P., Yang, M. Y., and Wang, C. J., "Mulberry extract inhibits the development of atherosclerosis in cholesterol-fed rabbits," Food Chemistry, Vol. 91, pp. 601-607 (2005).

6. Doi, K., Kojima, T., and Fujimoto, Y., "Mulberry leaf extract inhibits oxidative modification of rabbit and human low-density lipoprotein," Biological and Pharmaceutical Bulletin, Vol. 23, pp. 1066-1071 (2000).

7. Enkhmaa, B., Shiwaku, K., Katsube, T., Kitajima, K., Anuurad, E., Yamasaki, M., and Yamane, Y., "Mulberry (Morus alba L.) leaves and their major flavonol quercetin 3-(6-Malonylglucoside) attenuate atherosclerotic lesin," The Journal of Nutrition, Vol. 135, pp. 729-734 (2005).

8. Fiszman, S. M., Pons, M., and Damasio, M. H., "New parameter for instrumental texture profile analysis: instantaneous and retarded recoverable springiness," Journal of Texture Studies, Vol. 29, pp. 499-508 (1998).

9. Gerasopoulos, D., and Stavorulakis, G., "Quality characteristics of four mulberry (Morus sp.) cultivars in the area of China, Greece," Journal of the Science of Food and Agriculture, Vol. 73, pp. 261-264 (1997).

10. Giugliano, D., "Dietary antioxidants for cardiovascular prevention," Nutrition, Metabolism, and Cardiovascular Diseases, Vol. 10, pp. 38-44 (2000).

11. Hamed, M. G. E., Refai, F. Y., Hussein, M. F., and Elsamahy, S. K., "Effect of adding sweet potato flour to wheat flour on physical dough properties and baking," Cereal Chemistry, Vol. 50, pp. 140-146 (1973).

12. Katsube, T., Imawaka, N., Kawano, Y., Yamazaki, Y., Shiwaku, K., and Yamane, Y., "Antioxidant flavonol glycosides in mulberry (Morus alba L.) leaves isolated based on LDL antioxidant activity," Food Chemistry, Vol. 97, pp. 25-31 (2006).

13. Katsube, T., Tabata, H., Ohta, Y., Yamasaki, Y., Anuurad, E., and Shiwaku, K., "Screening for antioxidant activity in edible plant products:
Comparison of low-density lipoprotein oxidation assay, DPPH radical scavenging assay, and Folin-Ciocalteu assay," Journal of Agricultural and Food Chemistry, Vol. 52, pp. 2391-2396 (2004).

14. Khoo, U., Christianson, D. D., and Inglett, G. E., "Scanning and transmission microscopy of dough and bread," Bakers' Digest, Vol. 49, pp. 24-26 (1975).

15. Kim, H. J., Mortia, N., Lee, S. H., and Moon, K. D., "Scanning electron microscopic observations of dough and bread supplemented with Gastrodia elata Blume powder," Food Research International, Vol. 36, pp. 387-449 (2003).

16. Maki, Z., Tashiro, M., and Inamoto, H., "Stability of anthocyanin pigments of mulberry," Scientific Reports of the Kyoto Prefectural University, No. 32, pp. 23-28 (1981).

17. Matsuoka, T., Kimura, T. and Muraoka, N., "Research of the available constituents from mulberry tree," Tohoku Agricultural Research, Vol. 47, pp. 361-362 (1994).

18. Moss, R., "Dough microstructure as affected by the addition of cysteine, potassium bromate, and ascorbic acid," Cereal Sci. Today, Vol. 19, pp. 557-561 (1974).

19. Onogi, A., Osuwa, K., Yasuda, H., Sakai, A., Morita, H., and Itokawa, H., "Flavonol glycosides from the leaves of Morus alba L.," Shoya Kgaku Zasshi, Vol. 47, pp. 423-425 (1993).

20. Rojas, J. A., Rosell, C. M., Benedito-de-Barber, C., Perez-Munuera, I., and Liuch, M. A., "The baking process of wheat rolls followed by cryo-scanning electron microscopy," European Food Res. and Technol., Vol. 212, pp. 57-63 (2000).

21. Sangnark, A., and Noomhorm, A., "Effect of dietary fiber from sugarcane bagasse and sucrose ester on dough and bread properties," Lebensm.-Wiss. u-Technol., Vol.37, pp. 697-704 (2004).

22. SAS Institute, Inc., SAS User's Guide, Version 6 Edition, SAS Institute, Cary, North Carolina (1995).

23. Schneeman, B. O., "Dietary fiber and gastrointestinal function," Nutritional Research, Vol.18, pp. 625-632 (1998).

24. Vittadini, E., and Vodovotz, Y., "Changes in the physicochemical properties of wheat- and soy-containing breads during storage as studied by thermal analyses," Journal of Food Science, Vol.68, pp. 2022-2027 (2003).

25. Whalen, P. J., Bason, M.L., Booth, R. I., Walker, C. E., and Williams, P. J. "Measurement of extrusion effects by viscosity profile using the Rapid ViscoAnalyser," Cereal Foods World, Vol. 42, pp. 469-475 (1997).

26. Zhishen, J., Mengcheng, T., and Jianming, W., "The determination of flavonoid contents in mulberry and their scavenging effects in superoxide radicals," Food Chemistry, Vol. 64, pp. 555-559 (1999). 